\title{
Epidemiology of acute otitis in pediatric patients
}

\author{
Maddalena Perotti, Fabio Benzi, Luigia Ricagni, Luisa Pescetto, Roberto Bandettini \\ Laboratorio Centrale, Istituto G. Gaslini “Children Hospital”, Genova
}

Key words: Otitis, Children, Antibiotics

\section{Epidemiologia delle otiti acute in etá pediatrica}

\section{SUMMARY}

Introduction. Acute otitis is one of the most common pediatric infectious diseases that requires an accurate diagnosis in order to direct appropriate therapy to reduce the risk of complications. In this study pathogens collected from pediatric patients and their antibiotic susceptibility patterns were evaluated.

Methods. Between May 2009 and May 2010, 739 samples (swabs taken from nasopharynx in case of acute otitis media and/or from ears in case of acute external otitis, collected from 680 patients, suffering of otalgia, admitted to the emergency department of our Hospital were studied. The specimens were submitted for routine bacterial cultures and the susceptibility tests were performed according to Clinical Laboratory Standards. Nitrocefin was used to detect B-lactamase activity.

Results. 316 samples (42.8\%) of 739 were negative, 102 (13.8\%) were positive for Streptococcus pneumoniae, 97 (13.1\%) for Moraxella catarrhalis, 68 (9.2\%) for Haemophilus influenzae, 62 (8.4\%) for Pseudomonas aeruginosa, 49 (6.6\%) for Staphylococcus aureus, 36 (4.9\%) for Streptococcus pyogenes, 5 (0.7\%) for Gram negative and 4 (0.5\%) for Candida spp.

Antibiotic susceptibility tests showed that amikacin, ceftazidime, ciprofloxacin, imipenem, meropenem and piperacillin/tazobactam were active against all Gram negative strains isolated. We found one strain of MRSA. Of 102 Streptococcus pneumoniae, 5 (4.9\%) were penicillin resistant and 25 (24.5\%) were erythromycin resistant, showing the prevalence of constitutive phenotype (80\%). All M. catarrhalis strains were B-lactamase producers while all $H$. influenzae were ß-lactamase negatives.

Conclusions. The prevalent etiological agents in pediatric acute otitis are S. pneumoniae, M. catharralis, and $H$. influenzae, as reported in literature. In external acute otitis $P$. aeruginosa prevails in particular in summer.

\section{INTRODUZIONE}

Le otiti acute rappresentano una patologia molto comune in pediatria (1). Le otiti acute si differenziano in otiti esterne e otiti medie: le prime coinvolgono il condotto acustico esterno, le seconde l'orecchio medio (7). Le otiti esterne hanno una maggiore prevalenza in estate, a causa del clima caldo umido, e possono essere causate da una diminuzione dell'acidità del canale uditivo che promuove la crescita di batteri tra cui i principali sono Staphylococcus aureus, Pseudomonas aeruginosa e Streptococcus pyogenes (1). Le otiti medie, invece, sono spesso precedute da un'infezione delle prime vie respiratorie e sono causate dal passaggio attraverso le tube di Eustachio di batteri quali Haemophilus influenzae, Moraxella catarrhalis e $S$. pneumoniae $(3,5)$. La diagnosi e il trattamento di queste infezioni è fondamentale per prevenire la perdita di udito e altre gravi complicazioni quali mastoidite, batteriemia e meningite (4).

Scopo di questo studio è la valutazione degli agenti eziologici di otiti medie ed esterne di pazienti afferiti presso il nostro Istituto, la loro sensibilità agli antimicrobici e la stagionalità di queste infezioni.

\section{MATERIALI E METODI}

Da maggio 2009 a maggio 2010 sono stati studiati 680 pazienti di età compresa tra 0 e 14 anni (372 maschi e 308 femmine) afferenti presso il Pronto Soccorso di questo nosocomio pediatrico per otalgia.

Nel caso di otiti acute esterne (29.5\%) sono stati ricercati eventuali agenti eziologici tramite tamponi auricolari, mentre nel caso di otiti medie acute $(70.5 \%)$ attraverso indagini colturali della comunità batterica nasofaringea mediante tamponi nasofaringei eseguiti in profondità $(8,9)$.

I campioni (739) sono stati seminati su diversi terreni di coltura seguendo le linee guida (8): Agar Sangue, Agar Cioccolato, McConkey, Agar Sale Mannite e Chrom Agar Candida. Dalle indagini colturali sono stati esclusi i ceppi isolati consecutivamente e ripetutamente dallo stesso paziente.

Sui ceppi isolati è stata valutata la sensibilità agli antibiotici utilizzando il sistema automatizzato Phoenix (Becton Dickinson). I ceppi ATCC inse-

\section{Corresponding author: Maddalena Perotti}


riti nei saggi per il controllo di qualità sono stati: S. aureus 29213, P. aeruginosa 27853, S. pneumoniae 49619 e Escherichia coli 25922.

Per $H$. influenzae e $M$. catarrhalis è stata valutata la produzione di beta-lattamasi tramite il test del nitrocefin (Becton Dickinson).

\section{RISULTATI}

La Tabella 1 riporta i risultati ottenuti dalle semine dei campioni sui terreni appropriati. Dei 739 campioni studiati 316 sono risultati negativi, mentre la distibuzione dei 423 microoganismi isolati ha messo in evidenza, come atteso, che $S$. pneumoniae è stato il patogeno più frequentemente isolato $(24.1 \%)$ con M. catarrhalis $(22.9 \%)$.

$H$. influenzae $(16.1 \%)$ ha mostrato un'incidenza di poco superiore a quella di $P$. aeruginosa mentre gli altri germi sono stati ritrovati con frequenze oscillanti tra $11.6 \%$ (S. aureus) e $0.9 \%$ di Candida spp.

La distribuzione dei diversi agenti eziologici per patologia è rappresentata in Tabella 2.

Per quanto riguarda la valutazione dell'antibiotico sensibilità, tutti i ceppi Gram negativi isolati (62 P. aeruginosa, 2 Klebsiella oxytoca, 1 Escherichia coli, 1 Citrobacter spp., 1 Serratia marcescens) non hanno mostrato resistenza verso amikacina, ceftazidime, ciprofloxacina, imipenem, merope- nem e piperacillina/tazobactam; tutti i ceppi di $M$. catarrhalis sono risultati positivi al test del nitrocefin tutti i ceppi di $H$. influenzae sono risultati negativi al test del nitrocefin.

Considerando i Gram positivi, un ceppo di $S$. aureus è risultato meticillino resistente (Tabella 3), mentre i rimanenti 48 ceppi isolati hanno mostrato totale sensibilità alla clindamicina, ciprofloxacina e cotrimossazolo con incidenze elevate di ceppi inibiti per quanto riguarda la cefoxitina (98\%), eritromicina e gentamicina (94\%).

S. pyogenes (36), come atteso, sono risultati tutti sensibili alla penicillina (Tabella 3); per quanto riguarda i macrolidi ha prevalso il fenotipo costitutivo, mentre un solo isolato è stato identificato come veicolante un meccanismo di resistenza di tipo inducibile. Con riferimento ai 102 isolati di $S$. pneumoniae, $5(4.9 \%)$ sono risultati resistenti alla penicillina e $25(24.5 \%)$ resistenti all'eritromicina con il fenotipo costitutivo prevalente $(80 \%)$ (Tabella 4).

L'incidenza stagionale dei singoli patogeni è raffigurata in Figura I. Come è possibile vedere, tutti i patogeni mantengono un'incidenza che non supera il 10\% del totale, con l'eccezione di S. pneumoniae che si avvicina al $20 \%$ nel periodo maggiogiugno. Nei mesi estivi luglio e agosto P. aerugi-

Tabella I. Distribuzione degli agenti eziologici isolati

\begin{tabular}{|c|c|c|c|c|c|c|c|c|c|}
\hline & S.pneumoniae & M.catharralis & H.influenzae & P.aeruginosa & S.aureus & S.pyogenes & $\begin{array}{c}\text { *Altri Gram } \\
\text { negativi }\end{array}$ & $\begin{array}{c}\text { Candida } \\
\text { spp }\end{array}$ & Tot \\
\hline$\overline{N^{\circ}}$ & 102 & 97 & 68 & 62 & 49 & 36 & 5 & 4 & 423 \\
\hline$\overline{\%}$ & 24.1 & 22.9 & 16.1 & 14.7 & 11.6 & 8.5 & 1.2 & 0.9 & 100 \\
\hline
\end{tabular}

Tabella 2. Distribuzione agenti eziologici per patologia

\begin{tabular}{lcccccccc}
\hline Otite & S.pneumoniae & M.catharralis & H.influenzae & P.aeruginosa & S.aureus & S.pyogenes *Altri Gram & $\begin{array}{c}\text { Candida } \\
\text { negativi }\end{array}$ & spp \\
\hline Media & 102 & 97 & 68 & 0 & 0 & 30 & 0 & 0 \\
\hline Esterna & 0 & 0 & 0 & 62 & 49 & 6 & 5 & 4 \\
\hline
\end{tabular}

Tabella 3. Antibiotico-sensibilità di S. aureus (49)

\begin{tabular}{ccccccccc}
\hline & & Penicillina & Cefoxitina & Eritromicina & Clindamicina & Ciprofloxacina & Gentamicina & Cotrimossazolo \\
\hline \multirow{2}{*}{ S. aureus (\%) } & $\mathrm{S}$ & 0 & 98 & 94 & 100 & 100 & 94 & 100 \\
\cline { 2 - 10 } & $R$ & 100 & 2 & 6 & 0 & 0 & 6 & 0 \\
\hline
\end{tabular}

Tabella 4. Antibiotico-sensibilità di S. pyogenes (36) e S. pneumoniae (I02)

\begin{tabular}{ccccc}
\hline & & Penicillina & Clindamicina & Eritromicina \\
\hline S. pyogenes (\%) & S & 100 & 88 & 86 \\
\cline { 2 - 5 } & $\mathrm{R}$ & 0 & 12 & 14 \\
\hline S. pneumoniae (\%) & $\mathrm{S}$ & 95.1 & 80.4 & 75.5 \\
\cline { 2 - 5 } & $\mathrm{R}$ & 4.9 & 19.6 & 24.5 \\
\hline
\end{tabular}


nosa sfiora il 50\% degli isolati mentre $S$. aureus appare prossimo al 15\%. Nel bimestre settembreottobre solo $S$. pneumoniae supera il $20 \%$ e $M$. catarrhalis si attesta intorno al $16 \%$. Nei due mesi successivi $S$. pneumoniae è stato isolato con frequenza che sfiorava il $20 \%$, M. catarrhalis il $20 \%$ e $H$. influenzae intorno al $16 \%$. Nei primi due mesi dell'anno appare consistente la quota di $M$. catarrhalis che tende a raggiungere il $30 \%, S$. pneumoniae che supera il $20 \%$ e $H$. influenzae che fa registrare una percentuale di circa il $20 \%$ degli isolati. Il periodo marzo-aprile evidenzia che solo M. catarrhalis e $H$. influenzae sono stati capaci di superare il $10 \%$ degli isolati.

\section{CONCLUSIONI}

Dal nostro studio risulta che gli agenti eziologici prevalenti delle otiti medie sono $S$. pneumoniae, $H$. influenzae e $M$. catarrhalis, come da letteratura internazionale (2). Nei casi di otite esterna prevale $P$. aeruginosa che presenta una maggiore incidenza nei mesi estivi in quanto favorito dal caldo umido e dal ristagno di acqua nel canale uditivo in seguito all'attività balneare, seguito da $S$. aureus. Le micosi del condotto uditivo esterno sono risultate rare $(0.5 \%)$.

Per quanto riguarda la valutazione della sensibilità agli antibiotici dei ceppi isolati, i batteri Gram negativi non presentano resistenze ai più comuni antibiotici, e questo dato potrebbe trovare una spiegazione nel fatto che nell'ambito dei pazienti pediatrici la pressione selettiva è sicuramente inferiore a quella riscontrata negli adulti e che verosimilmente la distribuzione del farmaco a livello auricolare è inferiore ad altri distretti.

Confrontando i nostri dati sulla chemiosensibilità degli agenti eziologici di otiti medie con quelli raccolti nello studio Protekt Italia riguardo il paziente pediatrico, vediamo che la resistenza di $S$. pneumoniae a penicillina e macrolidi è inferiore alla media nazionale e lo stesso vale per $S$. pyogenes nei confronti dei macrolidi. Per quanto riguarda i ceppi di $H$. influenzae, che in questo studio sono risultati tutti non produttori di $\beta$-lattamasi, i dati discordano con la media nazionale che rileva un $24.7 \%$ di positività (6).

L'unico ceppo di MRSA isolato da un tampone auricolare appartiene a una paziente con otiti esterne recidivanti e quindi sottoposta a ripetute terapie antibiotiche sia topiche che sistemiche.

In definitiva la casistica di un anno di studio su queste patologie conferma i principali agenti eziologici e evidenzia una particolare sensibilità agli antibiotici dei ceppi isolati, che per quanto la sede anatomica non sia favorevole alla diffusione dei farmaci indica una probabile eradicazione del germe una volta che l'antibiotico viene a contatto con il patogeno.

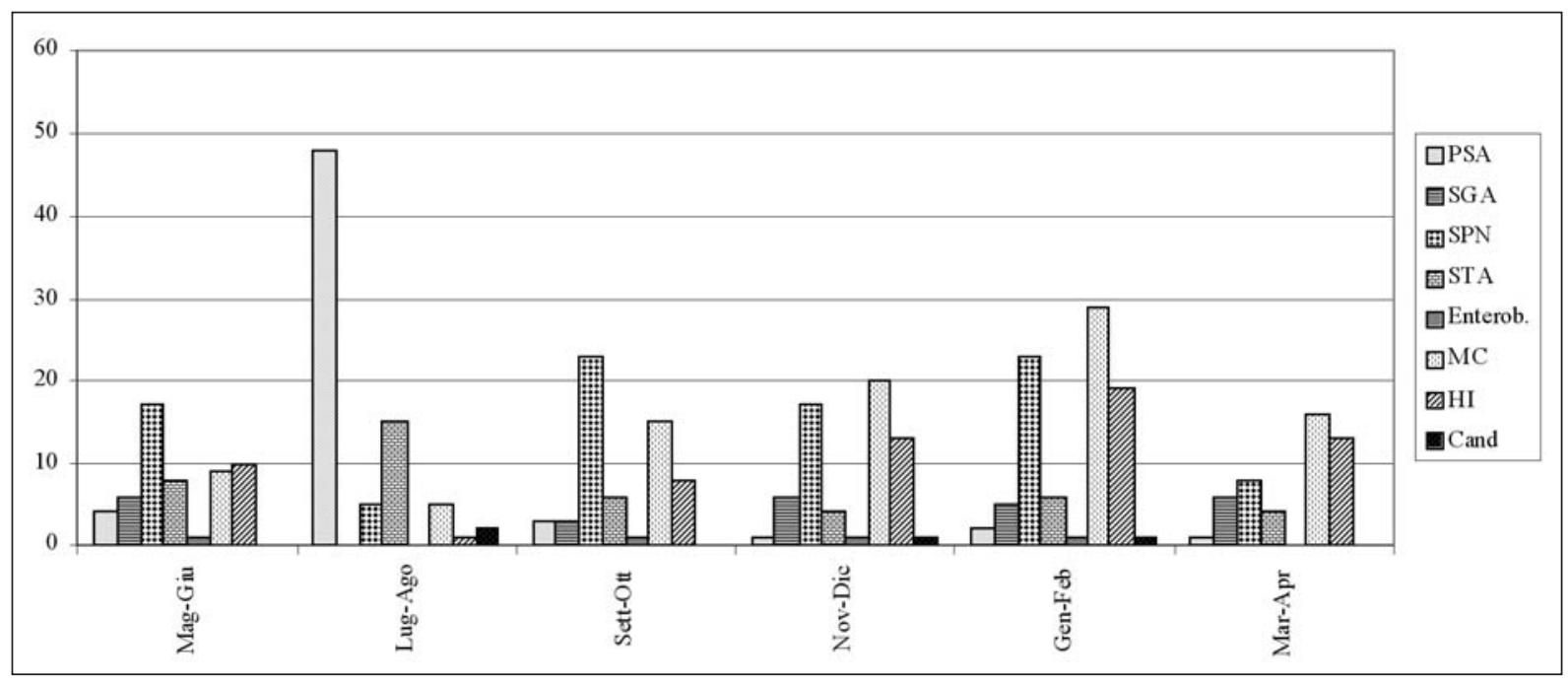

Figura I. Incidenza stagionale patogeni isolati

\section{BIBLIOGRAFIA}

1. Carlson L. Inside and out. A review of otitis in children. Adv Nurse Pract. 2007; 15 (5): 51-8.

2. Gené A, Garcia-Garcia JJ, Domingo A, Wienberg P, Palacin E. Etiology of acute otitis media in children's hospital and antibiotic sensitivity of the bacteria involved. Enferm Infect Microbiol Clin. 2004; 22 (7): $377-80$.
3. Gould JM, Matz PS. Otitis media. Pediatr Rev. 2010; 31 (3): 102-16.

4. Leibovitz E, Broides A, Greenberg D, Newman N. Current management of pediatric acute otitis media. Expert Rev Anti Infect Ther. 2010; 8 (2): 151-61.

5. Lynch JP 3rd, Zhanel GG. Streptococcus pneumoniae: epidemiology and risk factors, evolution of antimicrobial resistance, and impact of vaccines. Curr Opin Pulm Med. 2010; 16 (3): 217-25. Review 
6. Marchese A, Ardito F, Fadda G, et al. PROTEKT ITALIA: analisi dei risultati del terzo e ultimo anno di studio (2004) e considerazioni conclusive. GIMMOC 2005 (b); IX (2): 69-106.

7. Marchisio P, Bianchini S, Capaccio P, et al. Insights into infectious otitis media. Int $J$ Immunopathol Pharmacol. 2010; 23 (1 Suppl): 20-3.
8. Murray PR, et al. Manual of Clinical Microbiology $9^{\circ}$ Ed. American Society of Microbiology.

9. Syrjänen RK, Herva EE, Mäkelä PH, et al. The value of nasopharyngeal culture in predicting the etiology of acute otitis media in children less than two years of age. Pediatr Infect Dis J. 2006; 25 (11): 1032-6. 